names and (an innovation since Vol. 1) of German names used by Groth in his "Chemische Krystallographie". A new feature also is a page of "Brief Directions" for the experienced crystallographer, a copy of which can be obtained for insertion in Vol. 1.

Parts 2 and 3 of Vol. 2 contain the 3,572 crystal descriptions. These parts, and the tabular matter of Part 1, have been reproduced from typescript by offset litho. As in Part 2 of Vol. 1, references are given under each substance to a standard chemical or mineralogical work as well as to Groth ; some 125 substances not described by him are appended to Part 3. The descriptions include the matrix necessary to transform Groth's setting to the appropriate Barker setting, physical constants (including optical properties) and, where available, the spacings of the three strongest lines in powder photographs as given in the A.S.T.M. Index. During compilation it has been possible to correct a large number of errors, mainly in the calculations by original authors, which have been detected in Groth's descriptions. Quite aside from their use for purposes of identification, these two parts, together with Part 2 of Vol. 1, thus form a valuable compendium of up-to-date and accurate crystallographic information. Vol. 3 (dealing with crystals of the anorthic (triclinic) system) is in preparation. Its publication will complete an addition to crystallographic literature on which the committee and the editors are to be warmly congratulated.

$$
\text { F. C. Phillips }
$$

\section{ATOMIC STRUCTURES}

\section{The Calculation of Atomic Structures}

By Prof. Douglas R. Hartree. (Based on lectures given under the auspices of the William Pyle Philips Fund of Haverford College, 1955.) (Structure of Matter Series.) Pp. xiii +181. (New York : John Wiley and Sons, Inc. ; London: Chapman and Hall, Ltd., 1957.) 40s. net.

7 HIS book provides a simple account of methods used in calculations of atomic spectra. It has more the character of a guide to the subject rather than of an introduction, and is evidently intended for the specialist in a related field who becomes involved in atomic spectroscopy as an adjunct to his own work. It would also be of considerable value to the experimental spectroscopist who may be averse to advanced mathematics. The reader is assumed to have only an elementary know. ledge of mathematics and quantum theory, although he must be reasonably familiar with descriptive or experimental atomic spectroscopy, and if he is to make full use of the book he must not have an aversion to tables of functions.

There are several drawbacks to this elementary approach which make the book less satisfactory as an introduction than as a guide to the subject. The principal one is that it has forced the author to describe mainly the methods which were used during the earlier development of calculations on atomic spectra, rather than the more powerful and elegant methods which were developed later but which involve more difficult mathematics; in particular, the work of Racah is mentioned only in a passing refer. ence. Throughout, the author concentrates his attention on the methods of calculation rather than the general theory behind the methods ; for the budding theoretical physicist this is very much putting the cart before the horse, but there is no doubt that Prof. Hartree's consideration will be appreciated by others less mathematically inclined.

The first chapter introduces Schrödinger's equation for an electron in a central field, discusses the separable wave function approximation for a many-electron system and outlines the idea of the self-consistent field which was introduced by the author in 1927 and which forms the basis of all methods of computing atomic spectra. The second and third chapters describe the variation method for obtaining the energy of a quantum system, illustrating it by an application to helium and then to a many-electron system. The variation method is related to the Fock equations and the Hartree equations, and it is shown how the use of determinantal wave functions reduces the problem to the calculation of certain types of integral.

Chapter 4 gives a brief description of the elements of numerical methods, quoting formulæ without proof but with readily accessible references. The application of these methods to the solution of the equations for the energy of an atom with a complete group of electrons is described in the next chapter, and in Chapter 6 this is extended to atoms with incomplete groups of electrons. Methods of interpolation which are of use in guessing trial wave functions are discussed in Chapter 7.

The remaining Chapters 8, 9 and 10 deal somewhat briefly with more advanced topics concerning the energy differences of different states of ionization (Chapter 8), the use of the Dirac equation for the electron (Chapter 9), and recent improvements on the self-consistent field method of approximation.

R. J. EDEN

\section{STEREOCHEMISTRY}

Steric Effects in Organic Chemistry

Edited by Melvin S. Newman. Pp. vii +710. (New York: John Wiley and Sons, Inc.; London: Chapman and Hall, Ltd., 1956.) 100s. net.

TN the early development of electronic theories of 1 organic chemistry, much attention was focused on the influence of the polar effects of substituents on reactivity. To-day, now that this aspect of the subject has become extensively documented, increasingly more attention is being paid to stereochemical factors as they affect reaction-paths and mechanisms. Here must be considered not only bulk effects of substituents on the energy and entropy of activation, but also their effects in obstructing or enhancing the conjugative power of other substituents, the effects of varying the spatial relationships within the reacting molecule, and even the stereo-electronic considerations which determine whether bonds of particular types will be formed between pairs of atoms, and how strong these bonds will be.

Indeed, considering that organic reactivities are determined by the exact geometrical requirements and relationships of initial and transition states, a monograph on steric effects in organic chemistry has a very wide field to encompass. It is not surprising, therefore, that the work under review should be rather uneven in its coverage. In a number of chapters, equilibria and properties of ground-states of molecules are discussed; as in W. G. Dauben and K. S. Pitzer's account of conformational analysis ; G. S. Hammond's discussion of equilibria between 